\title{
The effect of epoch on the assessment of physical activity in Kenyan children.
}

\author{
Hannah Budd ${ }^{1}$, Robert Ojiambo ${ }^{1}$, Chris Easton ${ }^{1}$, Barry Fudge ${ }^{1}$, Kihumbu Thairu ${ }^{3}$, \\ John J. Reilly ${ }^{2}$, Yannis P. Pitsiladis ${ }^{1}$. \\ ${ }^{1}$ Faculty of Biomedical and Life Sciences, University of Glasgow, Glasgow, U.K. \\ ${ }^{2}$ Division of Developmental Medicine, University of Glasgow, Yorkhill Hospitals, \\ U.K. \\ ${ }^{3}$ Moi University Medical School, Eldoret, Kenya
}

Accelerometry is the preferred objective method for the assessment of sedentary and physical activity in children. Although the majority of studies utilise an epoch of $60 \mathrm{~s}$ for data collection, it may be more appropriate to use shorter epochs in children because their patterns of physical activity are highly intermittent (Bailey et al. Med Sci Sports Exerc 27: 1033-41, 1995). The limited existing evidence suggests that long epochs misclassify high activity as being of lower intensity, by averaging with bouts of lower intensity activity and sedentary behaviour within the same epoch (Nilsson et al. Pediatr Exerc Sci 14: 87-96, 2002).

PURPOSE: To compare measurements of sedentary and physical activity levels obtained using 1 and $60 \mathrm{~s}$ epochs in Kenyan children. METHODS: 27 children from the Arsi region of Kenya (Age 2-16) were assessed by uniaxial accelerometry (GT1M ActiGraph $^{\mathrm{TM}}$ ) for 3 consecutive days. The epoch duration was set at $1 \mathrm{~s}$ and data reintegrated to $60 \mathrm{~s}$ epoch using the device software and both files were edited to remove periods corresponding to sleep and periods containing $20 \mathrm{~min}$ or more of consecutive zeros. Activity levels were calculated using cut offs for sedentary, light and moderate to vigorous physical activity (MVPA) developed by Reilly et al. (Obes Res 11: 1155-8, 2003) and Puyau et al. (Obes Res 10: 150-7, 2002) for the 60 s epoch and by Pate et al. (Obesity 14: 2000-6, 2006) for the 1 s epoch. RESULTS: There were no significant differences in the average total counts per day and counts per min $(P=0.69$ and 0.59 respectively) between 1 and $60 \mathrm{~s}$ epochs. Furthermore, there were no differences between 1 and $60 \mathrm{~s}$ epochs for the average time spent engaging in sedentary activity ( 584 vs. $544 \mathrm{~min}, \mathrm{P}=0.28$ ). However, subjects spent a significantly greater period of time engaging in MVPA when calculated using the $1 \mathrm{~s}$ epoch compared to the $60 \mathrm{~s}$ epoch ( $57 \mathrm{vs.} 38 \mathrm{~min}, \mathrm{P}<0.01)$ and a significantly shorter period of time engaging in light activity (53 vs. $126 \mathrm{~min}, \mathrm{P}<0.01$ ). CONCLUSIONS: In the present study, a $60 \mathrm{~s}$ epoch misclassifies some MVPA as light intensity compared to the $1 \mathrm{~s}$ epoch and consequently significantly underestimates the daily duration of MVPA. Although the biological and clinical significance of this is unclear, a $1 \mathrm{~s}$ epoch may be more accurate for assessing physical activity in children. 